\title{
Lengthening Temporalis Myoplasty: Objective Outcomes and Site-Specific Quality-of-Life Assessment
}

\author{
Davide Thomas Panciera, MD', Claudio Sampieri, MD², \\ Alberto Deganello, MD, PhD $^{2}$, and Giovanni Danesi, MD'
}

No sponsorships or competing interests have been disclosed for this article.

\begin{abstract}
Objective. Evaluate outcomes of the lengthening temporalis myoplasty in facial reanimations.
\end{abstract}

Study Design. Case series with planned data collection.

Setting. Ospedali Riuniti, Bergamo, and AOUC Careggi, Florence, Italy.

Subjects and Methods. From 20II to 2016, II patients underwent lengthening temporalis myoplasty; demographic data were collected for each. Pre- and postoperative photographs and videos were recorded and used to measure the smile angle and the excursion of the oral commissure, according to the SMILE system (Scaled Measurements of Improvement in Lip Excursion). All patients were tested with the Facial Disability Index, and they also completed a questionnaire about the adherence to physiotherapy indications.

Results. All patients demonstrated a significant improvement in functional parameters and in quality of life. On the reanimated side, the mean $z$-line and a-value, measured when smiling, significantly improved in all patients: from $22.6 \mathrm{~mm}$ $(95 \% \mathrm{Cl}, 20.23-25.05)$ before surgery to $30.9 \mathrm{~mm}(95 \% \mathrm{Cl}$, 27.82-33.99) after surgery $(P<.001)$ and from $100.5^{\circ}(95 \%$ $\left.\mathrm{Cl}, 93.96^{\circ}-107.13^{\circ}\right)$ to $111.6^{\circ}\left(95 \% \mathrm{Cl}, 105.63^{\circ}-117.64^{\circ} ; P<\right.$ $.001)$, respectively. The mean postoperative dynamic gain, passing from rest to a full smile at the reanimated side, was $3.1 \mathrm{~mm}(95 \% \mathrm{Cl}, \mathrm{I} .30-4.88)$ for the $z$-line and $3.3^{\circ}(95 \% \mathrm{Cl}$, $1.26^{\circ}-5.29^{\circ}$ ) for the a-value. The Facial Disability Index score increased from a preoperative mean of 33.4 points (95\% Cl, 28.25-38.66) to 49.9 points $(95 \% \mathrm{Cl}, 47.2 \mathrm{I}-52.60)$ postoperatively $(P<.001)$.

Conclusions. The lengthening temporalis myoplasty can be successfully used for smile reanimation, with satisfying functional and quality-of-life outcomes.

\section{Keywords}

facial paralysis, facial reanimation, lengthening temporalis myoplasty, SMILE system, facial disability index, gracilis free flap
Received January 10, 20I7; revised May 9, 20I7; accepted June 6, 2017.

$\mathrm{F}$ acial paralysis is a complex condition with numerous consequences that negatively affect all aspects of a patient's life. Not only do the invalidating effects of facial paralysis concern self-image and social interactions with devastating psychological effects, but they also have important functional repercussions in eye protection, speech articulation, chewing, and swallowing.

The role of surgery in established peripheral facial palsy is driven by 2 fundamental goals: restore facial symmetry, both at rest and in active expression, and recover a spontaneous natural smile as much as possible. Evaluation of effectiveness in facial reanimation surgery is crucial for comparing and choosing the most suitable procedure among various options. The diversity of surgical procedures for facial reanimation and the difficulty in properly assessing the respective outcomes pose a challenge for surgeons, who have to choose the most appropriate treatment for the patient. Nevertheless, due to the lack of quality and uniformity in outcome data, there is a lack of consensus about the optimum surgical management of facial palsy, ${ }^{1}$ and the best practice is currently still based on experts' opinions.

Nowadays, the gold standard in the treatment of longstanding facial paralysis for lower facial reanimation is probably represented by the gracilis free muscle transfer; this procedure certainly demonstrated good results but it is a complex surgical technique requiring microvascular expertise, with a long learning curve. In 1997, Daniel Labbé ${ }^{2}$ described a promising technique named lengthening temporalis myoplasty, which presented the advantages of being a relatively simple and fast 1-step procedure. Despite these favorable characteristics, so far, in literature about 40

'Otolaryngology Unit of Azienda Ospedaliera Giovanni XXIII, Ospedali Riuniti, Bergamo, Italy

${ }^{2}$ Department of Surgery and Translational Medicine, University of Florence, Florence, Italy

Corresponding Author:

Alberto Deganello, MD, PhD, Department of Surgery and Translational Medicine, University of Florence, Largo Brambilla 3, 50134 Florence, Italy. Email: adeganello@hotmail.com 
publications deal with this procedure, and half of the bulk belongs to Labbé. To date, Labbé probably operated on $>170$ patients $^{3}$; nevertheless, just over 100 cases were reported by others worldwide, and among these non-Labbé series, only 6 publications reported $>10$ patients. A recent review article on this topic ${ }^{4}$ highlighted that the only functional results so far available for this technique belong to 53 cases from 4 articles. $^{5-8}$ The review therefore encouraged the publication of more studies reporting results based on validated assessment tools by a combination of objective measurements and subjective quality-of-life scales. The aim of the present work is to meet these requests to provide a double-dimension analysis of the outcome achievable with lengthening temporalis myoplasty.

\section{Patients and Methods}

A retrospective analysis of a case series with planned data collection was designed. The accrual of the study was supposed to be closed after the enrollment of the first 10 consecutive patients, but since the 10th and the 11th were operated almost simultaneously, we enrolled a further patient, resulting in a cohort of 11. All patients underwent lengthening temporalis myoplasty over a 6-year period from 2011 to 2016 at Papa Giovanni XXIII Hospital in Bergamo and at the Careggi University Hospital in Florence. Institutional review board approval was obtained by both institutions (Comitato Etico Provinciale di Bergamo and Comitato Etico Area Vasta Centro-AOUC Careggi).

\section{Surgical Procedure}

The preoperative evaluation is a fundamental moment: the determination of the type of smile, based on the unaffected side, will serve as guide to locate the main traction points at the nasolabial fold to identify the correct vectors of traction, as already described. ${ }^{9}$ These points must be marked to insert the tendon anchor points in the correct places during the operation. Surgery was performed as previously described by Labbé, ${ }^{2,9-11}$ Labbé described 2 variations of the technique: version 1 (V1) and version 2 (V2).

The V1 and V2 procedures start with the incision in the nasolabial fold, placement of the traction points based on the type of smile, and exposure of the coronoid process and temporalis tendon. Originally, for the V1, a bicoronal incision was described; however, we found that for both V1 and $\mathrm{V} 2$ procedures, a hemicoronal incision is sufficient.

In the V1 procedure, the temporal fascia is dissected until the orbital rim and the zygomatic arch. The arch is sectioned to access the infratemporal fossa, and the residual masseteric fibers are separated from the lateral surface of the temporalis muscle; at this point, the osteotomy of the coronoid process is performed from the temporal incision. All the insertions of the temporalis muscle are now incised, and the muscle is dissected off the temporal fossa and finally completely elevated, with attention paid not to damage the neurovascular pedicle.

In the V2 technique, the zygomatic arch is left intact, and the lateral surface of the temporal muscle, from the zygomatic arch to the lateral surface of the coronoid process, is freed from the masseteric fibers with blunt dissection under the arch. The temporalis muscle origins are incised only in the posterior and inferior attachments. The muscle belly is elevated from the temporal fossa, maintaining the anterior and superior insertions anchored to the bone, and the osteotomy of the coronoid process is performed through the nasolabial incision.

For both procedures, the coronoid process is retrieved through the nasolabial incision, the tendon is detached from the coronoid process and sutured to the previously placed anchor points. The temporalis muscle body is then resuspended and resutured at its origins to obtain the desired amount of labial suspension. In V1, the zygomatic arch is fixed.

For the 10 patients operated at Bergamo hospital, the V1 procedure was used, with the only modification being a hemicoronal incision instead of a bicoronal incision; the patient at the Florence university hospital underwent a V2 procedure. All patients had already received surgery for eye correction depending on the requirements; Table I shows demographic data, including age, sex, duration of palsy, etiology, and previous radiotherapy. Patients were instructed by a professional physiotherapist to perform specific exercises multiple times a day for 6 months.

\section{Outcome Analysis}

To obtain an objective outcome, pre- and postoperative (6 months) photographs and videos were used to measure the smile angle and the commissure excursion of every patient at rest and when attempting to make a full smile. These measures were recorded with the Scaled Measurements of Improvement in Lip Excursion (SMILE technique). ${ }^{12}$ With use of Photoshop (Adobe Systems Inc, San Jose, California), the iris white-to-white diameter was measured and divided by the standardized human average $(11.7 \mathrm{~mm})$ to produce a photograph-specific scaled vector, which was then applied to additional measurements in the same photograph to calculate facial dimension as follows. Commissure excursion (z-line), the distance from the lower vermilion midpoint to the oral commissure, and the smile angle (a-value), the angle between the z-line and the vertical midline (Figure I), were measured for both sides at rest and when smiling. The symmetry of the lower face was assessed for each patient: differences in SMILE values between the normal side and the affected side were calculated pre- and postoperatively, at rest and when smiling. The pre- and postoperative dynamic gain from rest to a full smile was also calculated for $\mathrm{z}$-lines and a-values.

The patients were asked to answer the Facial Disability Index ${ }^{13}$ questionnaire twice, preoperatively and 6 months after surgery, to assess subjective improvements in quality of life. We translated these questions to Italian until 2014, when a validated Italian version of the questionnaire was published $^{14}$ : the official version of the questionnaire did not differ from our previous translation.

Patients were also asked to fill in a questionnaire about their adherence to physiotherapy prescriptions (they had to 
Table I. Cohort Characteristics.

\begin{tabular}{|c|c|c|c|c|}
\hline $\begin{array}{l}\text { Patient No.: } \\
\text { Sex, Age (y) }\end{array}$ & Etiology and Previous RT & $\begin{array}{l}\text { Duration of } \\
\text { Palsy, mo }\end{array}$ & Previous Eye Surgery & Follow-up, mo \\
\hline I: F, 56 & Schwannoma VIII & 27 & Upper eyelid loading & 69 \\
\hline 2: $M, 70$ & Cholesteatoma & 12 & Upper eyelid loading & 49 \\
\hline 3: M, 6I & Parotid adenocarcinoma, previous RT & 30 & Upper eyelid loading & 7 \\
\hline 4: M, 70 & $\begin{array}{l}\text { Parotid adenoid cystic } \\
\text { carcinoma, previous RT }\end{array}$ & 11 & Upper eyelid loading & 14 \\
\hline 5: F, 53 & Schwannoma VII & 13 & $\begin{array}{l}\text { Upper eyelid loading, } \\
\text { lateral tarsorrhaphy }\end{array}$ & 49 \\
\hline 6: F, 5I & Schwannoma VIII & 27 & Upper eyelid loading & 6 \\
\hline 7: F, 36 & Schwannoma VIII & 34 & Lateral tarsorrhaphy & 23 \\
\hline 8: $M, 19$ & Teratoma of the petrous bone & 22 & Lateral tarsorrhaphy & 59 \\
\hline 9: F, 65 & Bell's palsy & 29 & Upper eyelid loading, lateral tarsorrhaphy & 42 \\
\hline I0: M, 57 & Cholesteatoma & 16 & Upper eyelid loading & 24 \\
\hline II: M, 80 & $\begin{array}{l}\text { Otitis media post-RT for } \\
\text { nasopharyngeal carcinoma }\end{array}$ & 32 & Brow suspension, lateral tarsorrhaphy & 8 \\
\hline
\end{tabular}

Abbreviations: F, female; $\mathrm{M}$, male; RT, radiotherapy.

practice several exercises every day for 6 months), to evaluate whether the rehabilitation could influence the outcome. The final total score could range from 0 to 10 points, calculated as follows: how many months they practiced the exercises ( 1 point for each month of practice, 6 points maximum), how many days per week $(0=0$ or 1 day a week, $1=2$ days, $2=3-6$ days, $3=$ every day), and use of

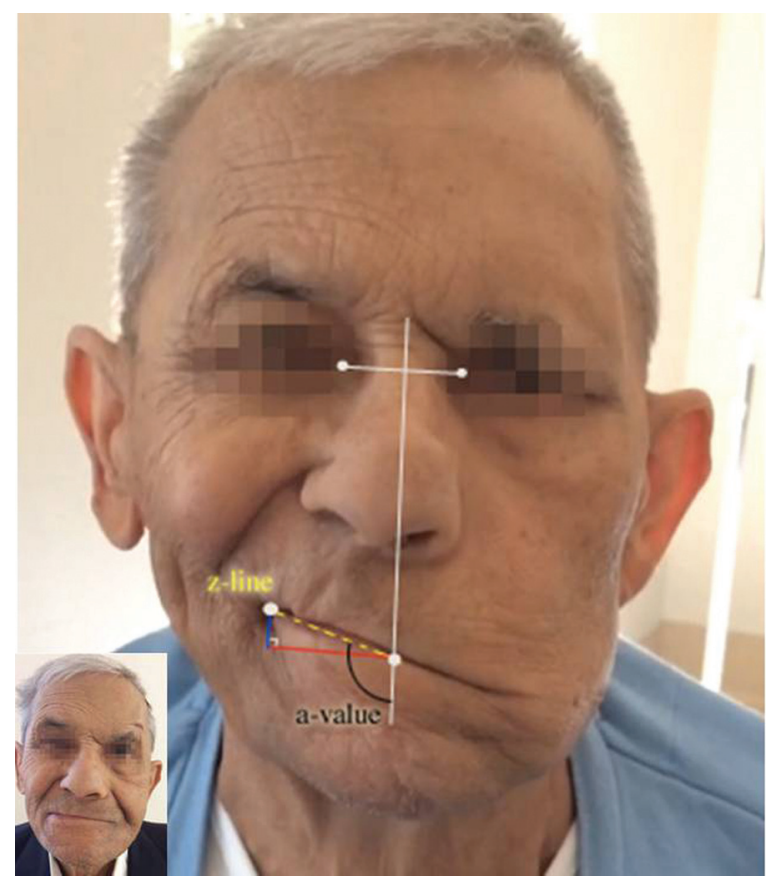

Figure I. Preoperative 80 -year-old smiling patient with SMILE measurements: the z-line represents the commissure excursion, and the a-value represents the smile angle. The small photo shows the postoperative result at full smile. SMILE, Scaled Measurements of Improvement in Lip Excursion. electrostimulations $(0=$ no, $1=$ yes $)$. A 0 or 1 score indicated a total lack of adherence; 2 or 3 , extremely poor adherence; 4 or 5 , poor adherence; 6 or 7 , medium adherence; 8 or 9 , good adherence; and 10 , full adherence.

\section{Statistical Analysis}

Mean values and standard deviations were calculated to compare pre- and postoperative parameters; the statistical significance of each observed difference was tested by a 2 tailed $t$ test.

We took into account the multiple-comparison problem that occurs when dealing simultaneously with a set of statistical inferences, ${ }^{15}$ and due to the low sample size, when 9 repeated comparisons are performed at a 5\% significance level, the experiment-wide significance level is given by $\alpha_{\text {TOT }}=1-(1-\alpha)^{9}=1-(1-0.05)^{9}=0.36975=37 \%$. Evidently, $\alpha_{\text {TOT }}=37 \%$ is not acceptable. We decided to halve the $\alpha_{\text {TOT }}$ to $18 \%$ so that in the end we considered $\alpha=$ $\alpha_{\text {TОT }} / 9=0.18 / 9=0.2=2 \%$, by applying the Bonferroni correction, ${ }^{16}$ and the same result was obtained via the Šidák $\operatorname{method}^{17}: \alpha=1-\left(1-\alpha_{\mathrm{TOT}}\right)^{1 / 9}=1-(1-0.18)^{1 / 9}=$ $0.02181 \asymp 0.02=2 \%$. Therefore, the statistical significance was set for $P<.02$. Finally, for each mean, 95\% CI was estimated to test that it was significantly different from the null value.

All statistical analysis was performed with STATA 13 (StataCorp, College Station, Texas).

\section{Results}

The mean operative time was 90 minutes; all procedures were carried out without complications; and all patients were discharged home within 5 days. One patient developed a hypertrophic scar at the nasolabial incision and underwent a second revision operation. 
Table 2. SMILE System Measurements. ${ }^{\text {a }}$

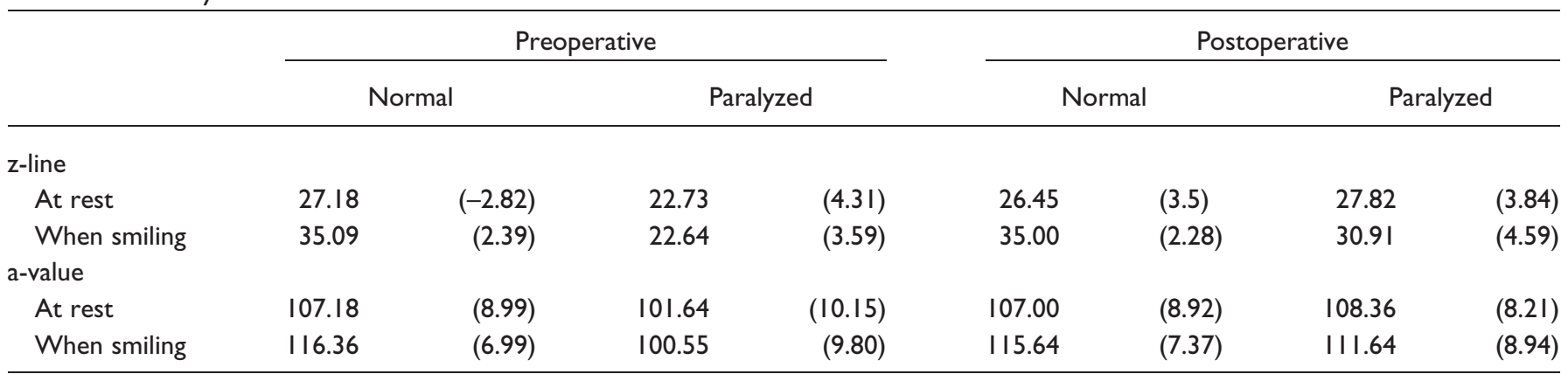

Abbreviation: SMILE, Scaled Measurements of Improvement in Lip Excursion.

${ }^{a}$ Values are presented as mean (SD).

Table 3. Comparisons of Pre- and Postoperative Outcomes: Results of the 2-tailed $t$ Tests.

\begin{tabular}{|c|c|c|c|c|c|}
\hline & \multicolumn{4}{|c|}{ Mean $(95 \% \mathrm{Cl})$} & \multirow[b]{2}{*}{$P$ Value } \\
\hline & \multicolumn{2}{|c|}{ Preoperative } & \multicolumn{2}{|r|}{ Postoperative } & \\
\hline \multicolumn{6}{|l|}{ z-line symmetry ${ }^{\mathrm{a}}$} \\
\hline At rest & 4.45 & (I.52 to 7.39$)$ & -1.36 & $(-3.12$ to 0.40$)$ & .0028 \\
\hline When smiling & 12.45 & (9.60 to $\mid 5.3 I)$ & 4.09 & $(0.98$ to 7.21$)$ & .0001 \\
\hline \multicolumn{6}{|l|}{ a-value symmetry ${ }^{\mathrm{b}}$} \\
\hline At rest & 5.55 & (2.57 to 8.53$)$ & -1.36 & $(-3.12$ to 0.40$)$ & .0016 \\
\hline When smiling & 15.82 & $(12.19$ to 19.45$)$ & 4.00 & (I.43 to 6.57$)$ & .0002 \\
\hline \multicolumn{6}{|c|}{ Dynamic gain of the paralyzed side } \\
\hline z-line ${ }^{c}$ & -0.09 & $(-1.01$ to 0.83$)$ & 3.09 & ( 1.30 to 4.88$)$ & .0048 \\
\hline a-value ${ }^{d}$ & -1.09 & $(-4.15$ to 1.96$)$ & 3.27 & ( 1.26 to 5.29$)$ & .0251 \\
\hline \multicolumn{6}{|c|}{ When smiling: paralyzed side } \\
\hline z-line & 22.64 & (20.23 to 25.05$)$ & 30.91 & (27.82 to 33.99$)$ & .0000 \\
\hline a-value & 100.55 & (93.96 to 107.13$)$ & 111.64 & (I05.63 to 117.64$)$ & .0001 \\
\hline Facial Disability Index & 33.45 & (28.25 to 38.66$)$ & 49.91 & $(47.21$ to 52.60$)$ & .0000 \\
\hline
\end{tabular}

${ }^{a} z$-line symmetry $=$ (normal side $z$-line $)-($ paralyzed side $z$-line $)$.

ba-value symmetry $=($ normal side a-value $)-($ paralyzed side a-value $)$.

${ }^{c} z$-line dynamic gain $=($ when smiling $z$-line $)-($ at rest $z$-line $)$.

$\mathrm{d}_{\mathrm{a}-\mathrm{value}}$ dynamic gain $=($ when smiling a-value $)-($ at rest a-value $)$.

Table 2 summarizes the results obtained with the SMILE system measurements, while Table 3 shows the comparisons between pre- and postoperative outcomes. On the reanimated side, the mean z-lines and the mean a-values, measured when smiling, significantly improved in all patients: from $22.64 \mathrm{~mm}(95 \% \mathrm{CI}, 20.32-25.05)$ before surgery to $30.91 \mathrm{~mm}(95 \% \mathrm{CI}, 27.82-33.99)$ after surgery $(P<$ $.001)$ and from $100.55^{\circ}\left(95 \% \mathrm{CI}, 93.96^{\circ}-107.13^{\circ}\right)$ to $111.64^{\circ}$ $\left(95 \%\right.$ CI, $\left.105.63^{\circ}-117.64^{\circ} ; P<.001\right)$, respectively. The differences between the normal side and the affected side in SMILE values significantly decreased after surgery, at rest and when smiling, resulting in a consistent improvement of facial symmetry. The mean postoperative z-line dynamic gain, passing from rest to a full smile, significantly improved after surgery at the reanimated side, reaching $3.09 \mathrm{~mm}$ (95\% CI, 1.30-4.88). Also the mean a-value dynamic gain improved, $3.27^{\circ}\left(95 \% \mathrm{CI}, 1.26^{\circ}-5.29^{\circ}\right)$; however, this improvement did not reach statistical significance $(P=.025)$.
Also the Facial Disability Index scores demonstrated a significant postoperative gain, reflecting an improvement in self-perceived quality of life with an increase from 33.4 points (95\% CI, 28.25-38.66) to 49.9 points (95\% CI, 47.21-52.60; $P<.001)$. The questionnaire analyzing adherence to physiotherapy prescriptions showed a medium score of 6.6 points ( $95 \%$ CI, 4.96-8.32), corresponding to medium adherence.

Figure 2 illustrates the preoperative condition and the postoperative outcome of patients who underwent a V1 lengthening temporalis myoplasty.

\section{Discussion}

The present work demonstrates a postoperative increase of the objective parameters measured with the SMILE technique and the subjective results recorded by the Facial Disability Index scores. It was not possible to perform direct comparisons with other studies, because there are no 


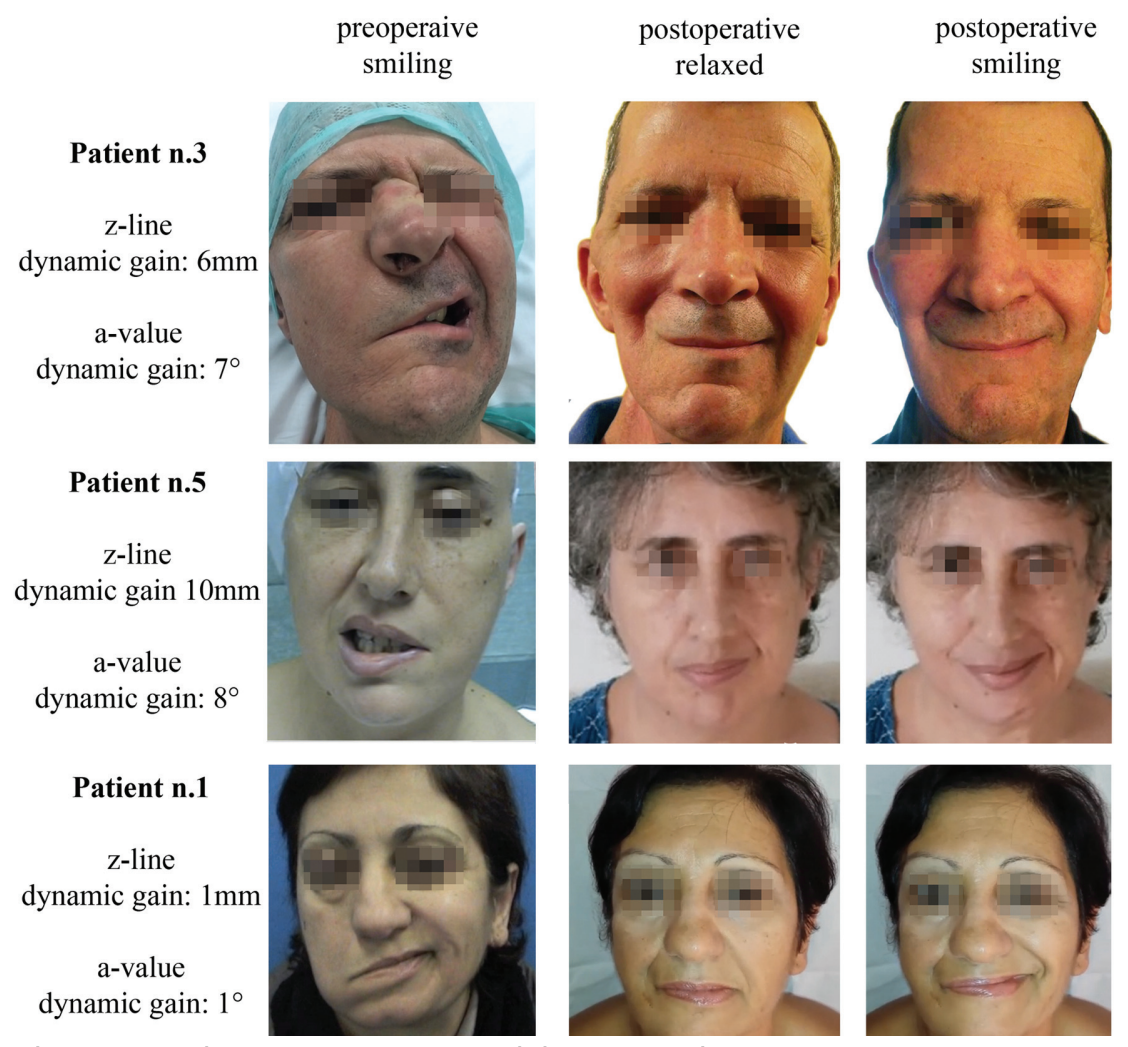

Figure 2. Preoperative smiling views and postoperative static and dynamic results.

publications about lengthening temporalis myoplasty with the SMILE system. Nonetheless, other studies on orthodromic temporalis tendon transfer procedures report quantitative data measured with various methods. Byrne et al, ${ }^{18}$ using measures similar to the SMILE system, reported a mean postoperative dynamic gain of $4.2 \mathrm{~mm}$ and a postoperative self-assessed quality-of-life score gain from 7.1 to 8.7 points out of 10 ; these results are in line with our findings. Scaglioni et al ${ }^{19}$ also demonstrated good dynamic results, in terms of commissural displacement and smile angle with improved symmetry, using a video-assisted method, unfortunately not directly comparable with our data.

Direct comparisons based on the SMILE technique can be made with other studies concerning facial reanimation surgery: Andrews et al, ${ }^{20}$ using the MIT3 technique, showed very similar values at full smile, including a mean postoperative z-line value of $31.2 \mathrm{~mm}$ and a mean postoperative a-value of $110.8^{\circ}$; Bray et $\mathrm{al}^{12}$ demonstrated better results using the gracilis free muscle transfer, with a mean postoperative z-line of $39 \mathrm{~mm}$ and a mean postoperative smile angle of $115^{\circ}$. When postoperative outcomes were evaluated, comparisons between the 2 sides of the face are extremely important. In fact, asymmetry, especially in the lower third of the face, generates negative feedback in the observer, negatively affecting patients' lives. ${ }^{21} \mathrm{~A}$ work on this topic by $\mathrm{Chu}$ et al ${ }^{22}$ highlighted that an oral commissure asymmetry $>3 \mathrm{~mm}$ could be perceived by the casual observer; therefore, it was suggested that surgery should aim to reduce asymmetry under this threshold. In our series the mean postoperative difference between normal and reanimated sides concerning z-line values met Chu's recommendations only at rest, $-1.36 \mathrm{~mm}(95 \% \mathrm{CI},-3.12$ to 0.40$)$, but it exceeded this threshold when smiling. Despite the significant improvement in facial symmetry, these data somehow reflect the low dynamic improvement produced by the Labbé procedure in our experience. In fact, in our study, the dynamic postoperative SMILE gains $(3.09 \mathrm{~mm})$ are lower than those reported in the literature with the gracilis free muscle transfer, which were between 6.5 and $7.8 \mathrm{~mm}$ for the z-line and $6.0^{\circ}$ to $8.7^{\circ}$ for the a-value..$^{23,24}$

Facial reanimation with the gracilis free flap and the transposition of regional muscles, such as lengthening temporalis myoplasty, share therapeutic indications, mainly in case of long-term facial paralysis. ${ }^{25} \mathrm{~A}$ recent review paper ${ }^{4}$ encouraged the publication of studies about the Labbé technique, reporting results by combination of objective measurements and subjective scales to obtain solid data that could be compared with those reported with other techniques. The purpose of the present work was to meet these recommendations, and despite the few patients enrolled, the results are encouraging: they indicate that the Labbé procedure is a valuable technique for an effective static correction, ensuring good postoperative facial symmetry, even though it produces less dynamic excursion gain when compared with the free gracilis muscle transfer. However, the goal of any treatment should always be centered on the patient's health, intended as a complete state of physical, 
mental, and social well-being; such a result cannot be expressed only through measurements of lengths or angles. The Facial Disability Index proved to be an optimal tool for evaluating the patient's subjective perception of the global outcome and its implication in daily life. The increase in the postoperative questionnaire scores, recorded in our series, bears witness to the improvement in quality of life perceived by patients. The Facial Disability Index also takes in consideration the eye-correlated problems, but in our cohort, eye surgery was performed in all cases before the smile reanimation procedure; therefore, the postoperative score improvement can only be correlated to the success of the Labbé technique. However, until further studies on lengthening temporalis myoplasty functional outcomes are published, the free gracilis muscle transfer remains the gold standard for long-term facial paralysis. Nevertheless, this technique is technically challenging, has a long learning curve, and is very demanding for the surgeon and, most of all, for the patient, and its global higher complexity can increase the rate of complications and medical costs. ${ }^{26,27}$

Previous studies on lengthening temporalis myoplasty highlight how adherence to the postoperative physiotherapy protocol is crucial to enhance the postoperative dynamic results. ${ }^{9,28}$ Perhaps, the lack of full adherence to the postoperative physiotherapy prescriptions, recorded in our series, might explain the low dynamic postoperative SMILE measurement gain; however, according to our data, physiotherapy does not seem to play a crucial role in the functional outcomes and quality of life. However, we acknowledge that the greater limitation of our study is represented by the small cohort of patients; therefore, our results suffer a lack of power to detect associations that may become statistically significant with greater numbers.

Giving the fact that these cases represent our first 11 patients undergoing the Labbé procedures, we acknowledge that in more experienced hands, the dynamic results could even be better than those recorded in our preliminary series. However, in our cohort, we did not observe a proportional improvement in the dynamic gain with the increasing experience; on the contrary, we noticed that the results were quite uniform: $10 \mathrm{~V} 1$ procedures were performed by D.T.P., with the only V2 by A.D., in 2 hospitals. No dynamic gain differences were noted between the first and last V1 cases, nor between the V2 case and the last V1 cases; therefore, our strong impression is that both procedures are rather reproducible and easy to perform by any surgeon with expertise in head and neck reconstructive surgery. It is not wise to exclude that, in the future, we might further progress by implementing nuances that come together with high numbers.

Despite being a long-described technique, the role of lengthening temporalis myoplasty is probably marginal in many hospitals, as testified by its scarce popularity in the literature. The establishment of a warehouse where multiple groups could enter their data for meta-analysis would help to better evaluate in a large scale the role and efficacy of this procedure in facial paralysis reanimation. The Facial
Disability Index is a very simple questionnaire to administer, while for the SMILE system, an automated, userfriendly, and free-to-use software program called FACEGram $^{29}$ was recently implemented, which allows one to easily and rapidly measure the SMILE parameters.

\section{Conclusion}

In our experience, the Labbe procedures demonstrated to be reliable options in facial paralysis surgery. Our preliminary results with lengthening temporalis myoplasty testify excellent static corrections paralleled by less brilliant dynamic outcomes, while we did not encounter major complications. We hope that these findings will stimulate further studies and multicenter collaborations to produce solid evidencebased algorithms on facial paralysis rehabilitation strategies.

\section{Acknowledgments}

We thank Laura Cagnoni and Vito Manni for their help in data collection.

\section{Author Contributions}

Davide Thomas Panciera, conception and design of the work, acquisition of data, drafting the article, final approval of the version to be published, agreement to be accountable for all aspects of the work in ensuring that questions related to the accuracy or integrity of any part of the work are appropriately investigated and resolved; Claudio Sampieri, conception and design of the work, analysis and interpretation of data, drafting the article and revising it critically for important intellectual content, final approval, agreement to be accountable for all aspects of the work in ensuring that questions related to the accuracy or integrity of any part of the work are appropriately investigated and resolved; Alberto Deganello, conception and design of the work, acquisition of data, analysis and interpretation of data, drafting the article and revising it critically for important intellectual content, final approval, agreement to be accountable for all aspects of the work in ensuring that questions related to the accuracy or integrity of any part of the work are appropriately investigated and resolved; Giovanni Danesi, conception and design of the work, analysis and interpretation of data, critically revising the article for important intellectual content, final approval, agreement to be accountable for all aspects of the work in ensuring that questions related to the accuracy or integrity of any part of the work are appropriately investigated and resolved.

\section{Disclosures}

Competing interests: None.

Sponsorships: None.

Funding source: None.

\section{References}

1. Hadlock T. Standard outcome measures in facial paralysis: getting on the same page. JAMA Facial Plast Surg. 2016;18: $85-86$.

2. Labbé D. Lengthening of temporalis myoplasty and reanimation of lips: technical notes [in French]. Ann Chir Plast Esthet. 1997;42:44-47.

3. Guerreschi P, Martinot-Duquennoy V, Labbé D. Re: "Lenghtening temporalis myoplasty: outcome and radiographic 
anatomical evaluation of length required." J Plast Reconstr Aesthet Surg. 2015;68:1468-1470.

4. Bos R, Reddy SG, Mommaerts MY. Lengthening temporalis myoplasty versus free muscle transfer with the gracilis flap for long-standing facial paralysis: a systematic review of outcomes. J Craniomaxillofac Surg. 2016;44:940-951.

5. Labbé D, Huault M. Lengthening temporalis myoplasty and lip reanimation. Plast Reconstr Surg. 2000;105:1289-1297.

6. Hayashi A, Labbé D, Natori Y, et al. Experience and anatomical study of modified lengthening temporalis myoplasty for established facial paralysis. J Plast Reconstr Aesthet Surg. 2015;68:63-70.

7. Veyssière A, Labbé D, Bénateau H. Lengthening temporalis myoplasty and facial paralysis from birth. J Plast Reconstr Aesthet Surg. 2015;68:312-320.

8. Gousheh J, Arasteh E. Treatment of facial paralysis: dynamic reanimation of spontaneous facial expression-apropos of 655 patients. Plast Reconstr Surg. 2011;128:693e-703e.

9. Labbè D, Bussu F, Iodice A. A comprehensive approach to long-standing facial paralysis based on lengthening temporalis myoplasty. Acta Otorhinolaryngol Ital. 2012;32:145-153.

10. Labbé D. Lenghtening temporalis myoplasty V.2. and lip reanimation [in French]. Ann Chir Plast Esthet. 2009;54:571-576.

11. Moubayed SP, Labbé D, Rahal A. Lengthening temporalis myoplasty for facial paralysis reanimation: an objective analysis of each surgical step. JAMA Facial Plast Surg. 2015;17: 179-182.

12. Bray D, Henstrom DK, Cheney ML, Hadlock TA. Assessing outcomes in facial reanimation: evaluation and validation of the SMILE system for measuring lip excursion during smiling. Arch Facial Plast Surg. 2010;12:352-354.

13. VanSwearingen JM, Brach JS. The Facial Disability Index: reliability and validity of a disability assessment instrument for disorders of the facial neuromuscular system. Phys Ther. 1996;76:1288-1298.

14. Pavese C, Cecini M, Camerino N, et al. Functional and social limitations after facial palsy: expanded and independent validation of the Italian version of the Facial Disability Index. Phys Ther. 2014;94:1327-1336.

15. Miller RG. Simultaneous Statistical Inference. 2nd ed. New York, NY: Springer Verlag; 1981.

16. Dunn OJ. Multiple comparisons among means. J Am Statist Assoc. 196;56:52-64.

17. Šidàk Z. Rectangular confidence region for the means of multivariate normal distributions. J Am Statist Assoc. 1967;62: 626-633.
18. Byrne PJ, Kim M, Boahene K, Millar J, Moe K. Temporalis tendon transfer as part of a comprehensive approach to facial reanimation. Arch Facial Plast Surg. 2007;9:234-241.

19. Scaglioni MF, Verdini F, Marchesini A, et al. Assessment of functional outcomes of temporalis muscle transfers for patients with longstanding facial paralysis. Head Neck. 2016;38: E1535-E1543.

20. Andrews P, Randhawa PS, Joseph J, et al. A prospective 4 year study of the objective and subjective outcomes of fifteen patients after dynamic facial reanimation surgery. Clin Otolaryngol. 2016;41:825-829.

21. Ishii L, Godoy A, Encarnacion CO, Byrne PJ, Boahene KDO, Ishii M. Not just another face in the crowd: society's perceptions of facial paralysis. Laryngoscope. 2012;122:533-538.

22. Chu EA, Farrag TY, Ishii LE, Byrne PJ. Threshold of visual perception of facial asymmetry in a facial paralysis model. Arch Facial Plast Surg. 2011;13:14-19.

23. Bhama PK, Weinberg JS, Lindsay RW, Hohman MH, Cheney ML, Hadlock TA. Objective outcomes analysis following microvascular gracilis transfer for facial reanimation: a review of 10 years' experience. JAMA Facial Plast Surg. 2014;16: $85-92$.

24. Vakharia KT, Henstrom D, Plotkin SR, Cheney M, Hadlock TA. Facial reanimation of patients with neurofibromatosis type 2. Neurosurgery. 2012;70:237-243.

25. Garcia RM, Hadlock TA, Klebuc MJ, Simpson RL, Zenn MR, Marcus JR. Contemporary solutions for the treatment of facial nerve paralysis. Plast Reconstr Surg. 2015;135:1025e-1046e.

26. Deganello A, Gitti G, Parrinello G, Muratori E, Larotonda G, Gallo O. Cost analysis in oral cavity and oropharyngeal reconstructions with microvascular and pedicled flaps. Acta Otorhinolaryngol Ital. 2013;33:380-387.

27. Garcia RM, Gosain AK, Zenn MR, Marcus JR. Early postoperative complications following gracilis free muscle transfer for facial reanimation: a systematic review and pooled data analysis. J Reconstr Microsurg. 2015;31:558-564.

28. Blanchin T, Martin F, Labbe D. Lengthening temporalis myoplasty: a new approach to facial rehabilitation with the "mirror-effect" method [in French]. Ann Chir Plast Esthet. 2013;58:632-637.

29. Hadlock TA, Urban LS. Toward a universal, automated facial measurement tool in facial reanimation. Arch Facial Plast Surg. 2012;14:277-282. 